\title{
adg2-1 represents a missense mutation in the ADPG pyrophosphorylase large subunit gene of Arabidopsis thaliana
}

\author{
Shue-Mei Wang', Becky Chu' ${ }^{2}$, Wei-Ling Lue ${ }^{2}$, Tien-Shin \\ $\mathrm{Yu}^{2}$, Klaus Eimert ${ }^{2}$ and Jychian Chen ${ }^{2}$ \\ ${ }^{1}$ Department of Botany, National Taiwan University, \\ Taipei, Taiwan and \\ 2Institute of Molecular Biology, Academia Sinica, Taipei, \\ Taiwan
}

\section{Summary}

Arabidopsis mutants affecting ADPG pyrophosphorylase (ADGase) activity can be divided into two complementation groups, adg1 and adg2. Previous biochemical studies of adg2-1 mutant indicated that mutant plants do not accumulate ADGase large subunit protein and that ADGase small subunits assemble as homotetramers. This suggested that the ADG2 gene may encode the large subunit of ADGase. In this paper, it is shown that adg2-1 mutant plants accumulate near wild-type levels of transcripts encoding both the large and small subunits of ADGase. However, by RFLP analysis and complementation of adg2-1 with the ADGase large subunit gene, we show that the adg2-1 mutant does represent a mutation of the ADGase large subunit gene. Sequence analysis of the adg2-1 allele revealed a missense mutation. The results therefore suggest either that the missense mutation affects the stability of the ADGase large subunit protein or that it prevents assembly of the large subunit into holoenzyme.

\section{Introduction}

ADP-glucose pyrophosphorylase (ATP: $\alpha$-glucose-1-phosphate adenylyl transferase, EC 2.7.7.27; ADGase) plays an important role in the synthesis of starch in both photosynthetic and non-photosynthetic plant tissues (Preiss, 1991). Within chloroplasts and amyloplasts, the enzyme catalyzes the synthesis of ADP-glucose and pyrophosphate from glucose-1-phosphate and ATP. ADP-glucose functions as the glucosyl donor for $\alpha$-glucan synthesis by various starch synthases (Preiss, 1991). Allosteric regulation of this

Received 17 October 1996; revised 2 January 1997; accepted 11 February 1997.

*For correspondence: Jychian Chen (fax +8862-7826085;

e-mail mbjchen@ccvax.sinica.edu.tw). enzyme by metabolites such as inorganic phosphate and 3-P-glycerate in plants and $5^{\prime}$-adenylate and fructose-1,6bisphosphate in bacteria plays a vital role in controlling the rate of starch synthesis (Preiss, 1988).

In bacteria and plants, the size of the native ADP-glucose pyrophosphorylase is similar, ranging from about 200 to $240 \mathrm{kDa}$ (Copeland and Preiss, 1981). The Escherichia coli enzyme is a homotetramer with a subunit size of about $50 \mathrm{kDa}$ (Haugen et al., 1976). By contrast, two different polypeptides of 51 and $54 \mathrm{kDa}$ copurified with the activity from spinach leaves (Copeland and Preiss, 1981; Morell et al., 1987). Other species such as Arabidopsis (Lin et al., 1988a,b), Chlamydomonas (Iglesias et al., 1994), wheat, rice, maize (Krishnan et al., 1986), and pea (Hylton and Smith, 1992) also contain immunologically related peptides of similar size. It was suggested that the native ADGase of plants was formed as heterotetramer with two large and two small subunits (Iglesias et al., 1995; Smith-White and Preiss, 1992). However, the assembly process has not been well characterized.

Mutants with low ADGase activity exhibit decreased starch accumulation. Such decreases are observed in leaves of the Arabidopsis mutants adg1 (Lin et al., 1988a) and adg2 (Lin et al., 1988b), in kernels of the maize mutants shrunken2 (sh2) and brittle2 (bt2) (Weaver et al., 1972), in seeds of the pea mutant $r b$ (Smith et al., 1989), and in tubers of potato plants expressing an antisense construct of an ADGase small subunit gene (Muller-Rober et al., 1992).

Arabidopsis mutants affecting ADGase activity can be divided into two complementation groups, adg1 and adg2. The adg2 mutant accumulates no ADGase large subunits and as such exhibits $5 \%$ of wild-type ADGase activity and $40 \%$ of wild-type starch levels in leaves. This mutant therefore demonstrates that the $54 \mathrm{kDa}$ large subunit protein is required for efficient ADGase function (Lin et al., 1988b). However, since adg2 mutant plants accumulate no ADGase large subunit protein yet have some ADGase activity, it appears that the homotetramer of small subunits is at least partially functional (Li and Preiss, 1992).

The adg 1-1 mutant is conditioned by a monogenic recessive mutation. Mutant plants accumulate less than $2 \%$ of wild-type starch levels and have no detectable ADGase activity in the leaves (Lin et al., 1988a). By Western blot analysis, the adg1-1 mutant was shown to lack both the large and small subunit proteins of ADGase. Based on 
these results, a hypothesis was proposed that the adg 1-1 is a regulatory gene affecting both large and small subunit gene expression (Lin et al., 1988a).

In this paper, we show that $a d g 2$ represents a missense mutation in the large subunit gene of ADGase. Our results suggest that the large subunit protein is not stable in the chloroplast unless it is assembled as a heterotetramer.

\section{Results}

\section{Characterization of cDNA and genomic clones of ADGase} large subunit

To examine ADGase small and large subunit gene expres sion in adg2 mutant plants, we characterized previously isolated ADGase cDNA clones (Villand et al., 1993) and isolated the ADGase genomic clones. Four cDNA clones of ADGase, including one small subunit (APS) and three large subunits (APL1, APL2 and APL3), were isolated by PCR with two primers corresponding to conserved domains and RNA isolated from whole plants (Villand et al., 1993). Two cDNA clones corresponding to the small (APS1A) and one of the large subunits (APL1A) were isolated from a leaf (Columbia ecotype) cDNA library using the PCR isolated CDNAs (APS and APL1) as probes (Villand et al., 1993). We sequenced the APL1A cDNA clone and found that it contained an insert of $1.67 \mathrm{~kb}$ with an open reading frame encoding the entire ADGase large subunit protein (Figure 1). To isolate genomic clones of the ADGase large subunit, we screened a genomic library (Landsberg erecta ecotype) with the APL1A cDNA probe. By restriction enzyme mapping and partial sequence analysis of the genomic clones, we showed that one of the genomic clones (APL14B) included the complete ADGase large subunit transcription unit plus $3.2 \mathrm{~kb}$ of $5^{\prime}$ upstream and $1 \mathrm{~kb}$ of $3^{\prime}$ downstream sequence. The restriction map and orientation of the transcription unit are shown in Figure 1.

\section{Northern blot analysis}

We examined ADGase transcript accumulation in leaves of wild-type and ADGase mutant plants by Northern blot analysis. Total RNA was isolated from leaves of plants grown in continuous light, electrophoresed on formaldehyde-agarose gels, transferred to a nytran membrane, and then probed with the small subunit and large subunit cDNAs of ADGase. As shown in Figure 2, transcripts encoding the ADGase large and small subunits were detected at similar levels in wild-type, adg1-1 and adg2-1 mutants. Since adg2-1 mutants do not accumulate ADGase large subunit protein but do accumulate large subunit transcripts, we reasoned that the adg2-1 mutation must perturb either the translation or post-translational processing of ADGase large subunit protein.

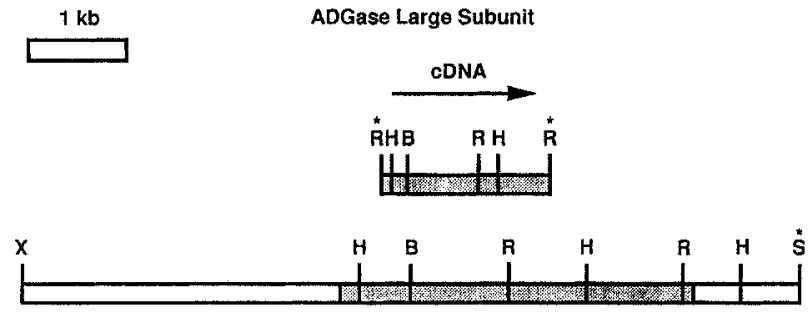

Figure 1. Restriction maps of Arabidopsis large subunit cDNA and genomic clones.

The transcription orientation of Arabidopsis ADGase large subunit is indicated with an arrow along with the restriction enzyme maps of large subunit cDNA and genomic clones: $X$ (Xbal), H (Hindlll), B (BamHI), $R$ $(E c o R I), S(S s t))$. Sites labeled with an asterisk are present in the vectors; shaded boxes represent transcribed regions.
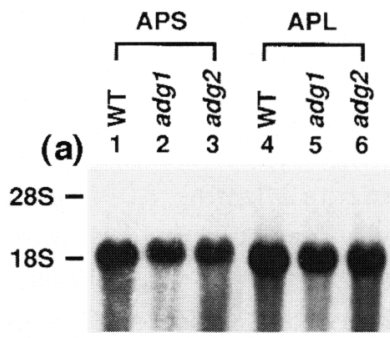

(b)
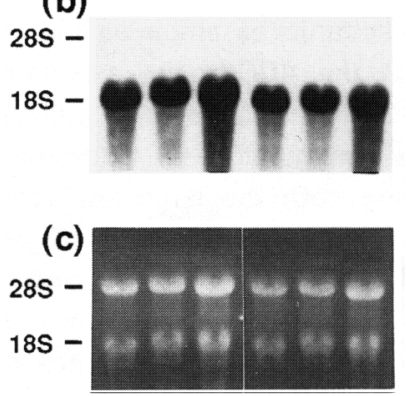

Figure 2. Northern blot analysis of small subunit and large subunit transcripts of ADGase.

(a) Northern blots of total leaf RNA $(20 \mu \mathrm{g})$ isolated from wild-type, adg $1-1$ and adg2-1 plants probed with radioactively labeled small subunit cDNA (APS) and large subunit cDNA (APL).

(b) After removal of the hybridization signals of small and large subunit probes, the same RNA blots as shown in (Figure 2a) were hybridized with a tubulin cDNA probe.

(c) The RNA gels were stained with ethidium bromide, showing that a similar amount of RNA was loaded.

\section{RFLP and DNA sequence analyses of adg2-1}

We mapped the ADGase large subunit gene by RFLP analysis. RFLP was identified by digesting genomic DNA of recombinant inbred lines (Lister and Dean, 1993) with Clal and probing with the APL1A CDNA. The data were analyzed using the JoINMAP computer program (Stam, 1993), indicating that the gene is located at chromosome 5-18.9 cM. To test whether the adg2 mutation is linked to the ADGase large subunit, we carried out RFLP analysis with selfed $\mathrm{F}_{2}$ progenies of adg2-1 (Col) crossed with the wild-type (Ler). In a population of 60 adg2-1 homozygous plants, which were identified by assaying for ADGase 
(a)

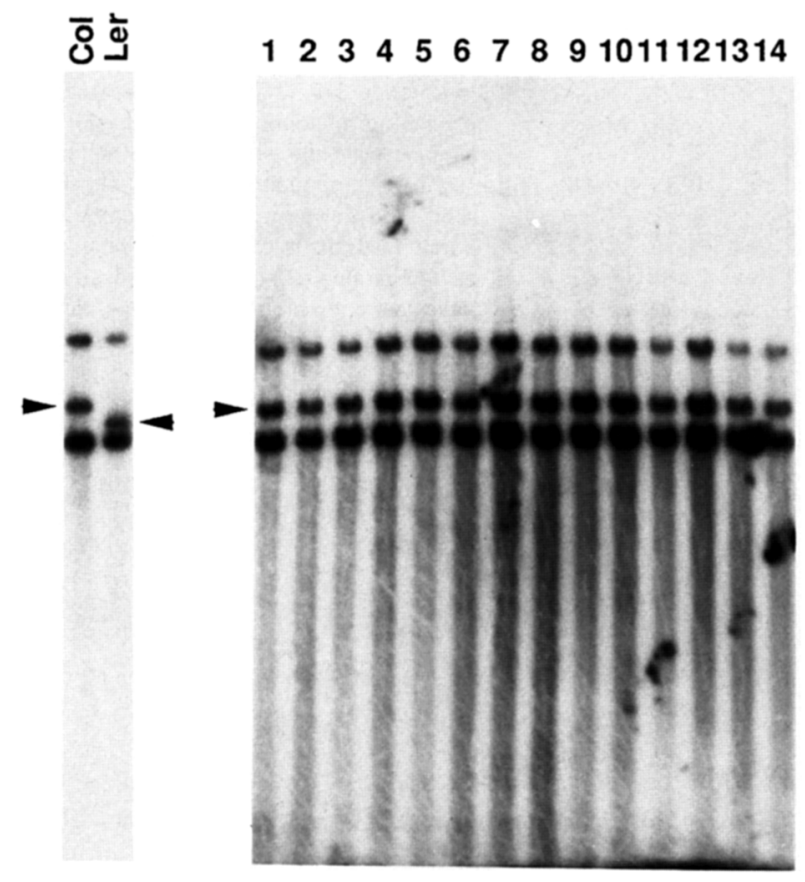

Figure 3. RFLP analysis of $F_{2}$ plants of adg2-7 (Col) $\times A D G 2$ (Ler). (a) Genomic DNAs $(2 \mu \mathrm{g})$ isolated from wild-type Columbia and Landsberg ecotypes were digested with Clal, Southern-blotted and probed with labeled ADGase large subunit cDNA. A polymorphism was detected.

(b) Southern blot analysis of genomic DNA isolated from $F_{2}$ progenies of adg2-1 (Col) $\times$ ADG2 (Ler). DNA samples derived from plants with the low leaf starch phenotype of adg2- (lanes 1-13) and from wild-type (Columbia) plants (lane 14) were digested with Clal, Southern-blotted, and probed with ADGase large subunit cDNA.

activity and leaf starch content, the APL1A Col band cosegregated with the low starch phenotype (Figure 3). This result strongly suggested that $a d g 2-1$ is a mutation in the ADGase large subunit gene.

We validated this suggestion by cloning and sequencing the adg2-1 cDNA. Primers were designed to the coding region of the gene. Reverse-transcribed cDNAs were synthesized from mRNA isolated from wild-type (coh) and adg2-1 (col) plants, amplified by PCR, cloned and sequenced. Multiple clones were sequenced from independent PCR reactions. The sequence data indicated that, in the adg2-1 mutant, there was a single nucleotide mutation at position 366 ( $G$ to $A$ ) that caused a glycine to glutamic acid (G118E) transition (Figure 4). The sequence around position 366 , which is downstream of the predicted transit peptide, is conserved in all other known ADGase large subunit genes (Smith-White and Preiss, 1992). To confirm that the mutant protein can be imported into chloroplasts, we synthesized radiolabeled proteins of the wild-type and the mutant ADGase large subunits through in vitro transcription and translation. Import assay with isolated pea chloroplasts indicated that the mutant ADGase large subunit protein was imported into chloroplasts and processed to the mature size (data not shown).

Complementation of adg2-1 mutant by transforming with the wild-type gene

To prove further that adg2-1 is an ADGase large subunit mutation, we transformed adg2-1 mutant plants with the wild-type genomic clone of the ADGase large subunit gene. The wild-type APL1 gene (an 8-kb Xbal-Sal fragment) was inserted into $\mathrm{pBin} 19$ and transformed into Agrobacterium tumefaciens C58 rif. adg2-1 mutants were transformed by vacuum infiltration, and 10 independent kanamycin resistant transformants were selected from the $T_{1}$ seeds. The kanamycin resistant transformants accumulated wildtype levels of starch. The $T_{1}$ plants were selfed, and the $T_{2}$ plants were scored for kanamycin resistance and assayed for ADGase activity and leaf starch content. Southern blot analysis indicated that transformants that exhibited kanamycin resistance, wild-type levels of ADGase activity and leaf starch, carried a single copy or multiple copies of the wild-type ADGase large subunit gene (Figure 5). In contrast, kanamycin sensitive plants showed the original adg2-1 phenotype. This result confirms that the adg2-1 mutant phenotype is caused by a mutation in the ADGase large subunit gene.

\section{Discussion}

Previous studies have shown that leaves of adg2-1 mutant plants accumulate no detectable ADGase large subunit protein (Lin et al., 1988b). By genetic and molecular analyses, we have shown that adg2-1 represents a missense mutation in the ADGase large subunit gene. A point mutation ( $G$ to $A$ ) at position 366 results in a glycine to glutamic acid (G118E) transition in the peptide sequence. Because the mutation site is not in the predicted transit peptide, the mutated nascent protein is transported into the chloroplast. However, since no large subunit protein is detected in adg2-1 mutant plants, the mutated protein must not be stable. It is possible that the mutated protein shows increased sensitivity to proteases and is thus degraded before it is assembled into the holoenzyme. Alternatively, the adg2-1 mutation may prevent the large subunit from being assembled into the holoenzyme, and unassembled large subunits may be rapidly degraded. Support for the latter hypothesis comes from analysis of the adg 1 mutant. adg1 represents a mutation in the ADGase small subunit gene (Chen, unpublished results). In adg 1 mutant plants, neither the small nor large subunit proteins accumulate, despite the fact that adg1 mutants have a normal large subunit gene (Lin et al., 1988a). This suggests that the assembly of large subunits into the holoenzyme is essential for stability. 
nucleotide \#335

Ara $\operatorname{adg} 2-1$

Ara LS

AAA CGC CGC GCC AAG CCT GCC GTT CCT ATC GGG

A

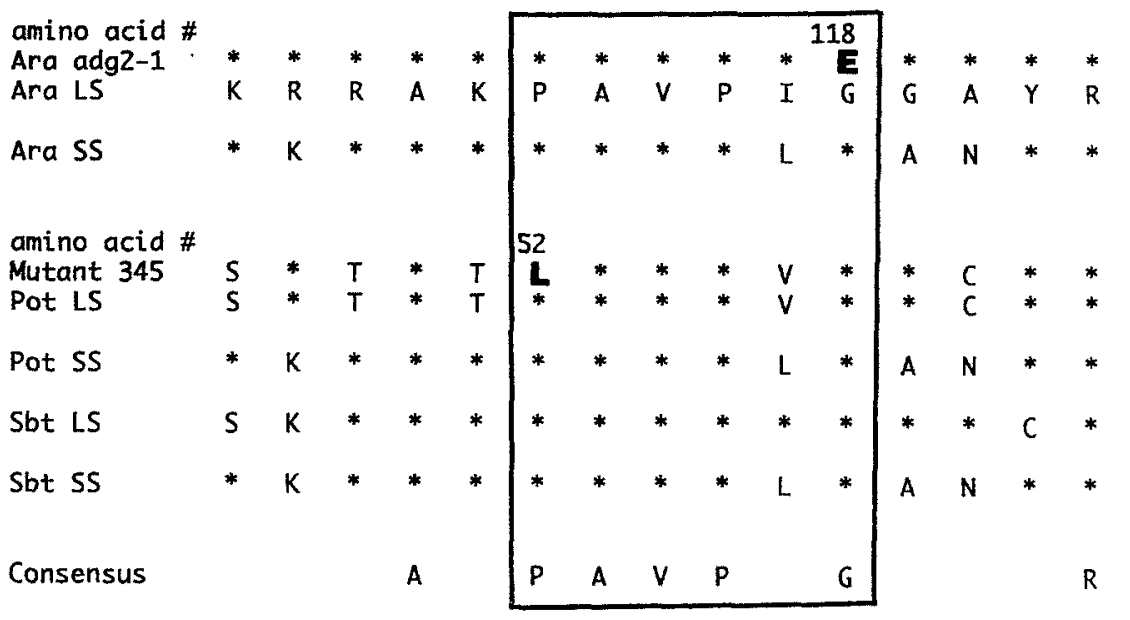

Figure 4. Sequence alignment around the missense mutation site of adg2-1.

The DNA and protein sequence of ADGase around the missense mutation site (nucleotide $366, G$ to $A$ and amino acid 118 glycine to glutamic acid) of adg2-1 were aligned with the small and large subunit sequences of potato (Pot) and sugar beet (Sbt), and the large subunit mutant 345 of potato (with the missense mutation at amino acid 52 proline to leucine). The boxed region indicates the proposed domain for the interaction of small and large subunits of ADGase. adg2 Transformant

T2 plants

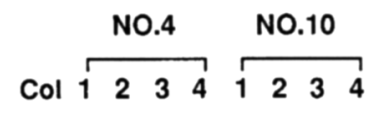

ADGase activity $\quad+++--++-$

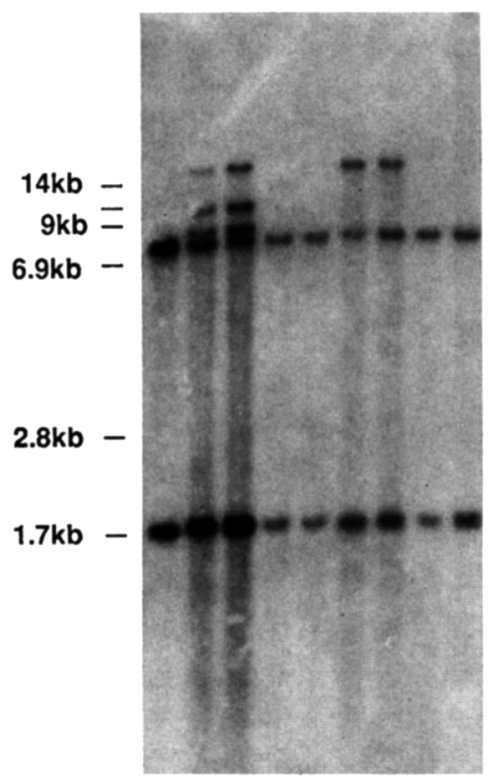

Figure 5. Southern blot analysis of two transgenic lines of adg2-1 carrying the transformed wild-type APL gene.

$T_{2}$ progenies of two representative transgenic lines (Nos 4 and 10) that cosegregated for kanamycin resistance, wild-type level leaf starch, and wildtype level leaf ADGase activity ( + ) (lanes 1-2); and for kanamycin sensitivity, low leaf starch, and low leaf ADGase activity phenotypes (-) (lanes 3-4). Genomic DNA was isolated from the leaves of wild-type (Columbia ecotype) and transgenic plants, digested with EcoRI, Southern blotted, and probed with ADGase large subunit CDNA. The results showed that transgenic plants with a wild-type phenotype carried the transformed wild-type ADGase large subunit gene.
While the adg2-1 allele appears to disrupt the assembly of large and small subunit ADGase proteins, other alleles may exist that affect the catalytic or allosteric regulatory domains of the enzyme. Such mutations permit the large subunit to be incorporated into the holoenzyme; however, holoenzyme activity is perturbed. This type of mutant has been identified by in vitro mutagenesis of the potato large subunit gene expressed in E. coli (Greene et al., 1996). One of these mutants (345) is deficient in glycogen synthesis but possesses ADGase enzyme activity comparable to wildtype as assayed in vitro. Like adg2-1, mutant 345 is a missense mutation of the ADGase large subunit gene. In mutant 345 , leucine exists at position 52 rather than proline (P52L). Position 52 is five amino acids upstream of the altered amino acid in adg2-1 (Figure 4). Significantly, the missense mutation of mutant 345 affects the interaction of the ADGase enzyme and its activator but does not affect the assembly of ADGase. Two suppressor mutants of mutant 345 that restore normal allosteric properties of ADGase have been isolated (Greene et al., 1996). Both mutants have the same $P 52 L$ missense mutation within the ADGase small subunit, suggesting that the allosteric domain of the heterotetramer holoenzyme is formed by the interaction of both the large and small subunits. Because the adg2-1 mutation alters the large subunit protein sequence only five amino acids downstream of that affected in mutant 345 , it is likely that the domain shown in Figure 4 is involved in the interaction of large and small subunits. This suggestion is further supported by the fact that the glycine at position 57 is conserved in all known ADGase sequences (Smith-White and Preiss, 1992). As such, the domain shown in Figure 4 is probably important for both allosteric regulation and holoenzyme assembly.

In Arabidopsis, ADGase large subunit genes exist as a small gene family with three members (Villand et al., 1993). 
Our complementation test suggested that the ADGase large subunit mRNA that accumulates in leaves is primarily transcribed from the $A D G 2$ gene. The expression profiles of the other two copies of the ADGase large subunit genes remain to be determined. It would be interesting to ascertain whether overexpression of the other two genes could functionally complement the adg2 mutation.

\section{Experimental procedures}

\section{Plant materials and growth conditions}

adg1-1, adg2-1 and recombinant inbred lines (Lister and Dean, 1993) were obtained from the Arabidopsis Biological Resource Center (ABRC) at Ohio State and Nottingham Arabidopsis Stock Center (NASC). Plants were grown in soil at $23^{\circ} \mathrm{C}$ under c. 5000 Lx continuous fluorescent light.

\section{Mutant screening}

Mutant screening was carried out as described by Caspar et al. (1985). Plants were screened first after they developed four to six true leaves. Leaf pieces were cut out, depigmented in $95 \%$ ethanol or methanol and stained with $\mathrm{I}_{2}-\mathrm{KJ}(5.7 \mathrm{mM}$ iodine and $43.4 \mathrm{mM}$ potassium iodide in $0.2 \mathrm{~N} \mathrm{HCl}$ ).

\section{Mapping}

RFLP mapping was performed according to Chang et al. (1988) and Nam et al. (1989), and the data were analyzed using the JoinMaP computer program (Stam, 1993).

\section{Native gel assay}

The ADGase gel assays were carried out according to Lin et al. (1988a) using $7.5 \%$ polyacrylamide.

\section{Isolation of genomic and CDNA clones}

Arabidopsis ADGase small subunit cDNA clone ALPC10 (APS1A) and large subunit CDNA clone ALPA4 (APL1A), both clones containing $1.7-\mathrm{kb}$ inserts in pBluescript vector (Stratagene), were isolated from a leaf cDNA library by screening with APS and APL1 probes respectively (Villand et al., 1993). APS, APL1, APS1A and APL1A plasmids were kindly supplied by Dr Villand. To isolate the corresponding genomic clones of ADGase large subunit, a genomic library of Arabidopsis Ler ecotype iconstructed as partially digested $M b o l$ genomic DNA fragments cloned into the Xhol site of lambda Fix vector, supplied by ABRC) was screened with the APL1A cDNA insert. An 8-kb Xbal-Sall fragment of the large subunit genomic lambda clone was further subcloned into pBluescript SK $^{+}$.

\section{General molecular analysis}

Standard cloning, Southern blot and Northern blot techniques were used as described by Sambrook et al. (1989). DNA sequencing was performed with double-stranded plasmids, using Sequenase (United States Biochemical).

\section{Complementation of ADGase mutants with wild-type genes by Agrobacterial transformation}

An $8 \mathrm{~kb} X b a l-S a l$ fragment of the ADGase large subunit genomic clone was cloned into $X b a l-S a h$ sites of $p B i n 19$ and denoted pBin 19-APL. The resulting T-DNA binary constructs were transformed into Agrobacterium tumefaciens pGV2260/C58 rif strain. adg2-1 plants were transformed with pBin19-APL by vacuum infiltration (Bechtold et al., 1993). Kanamycin-resistant transformants were selected by germinating seeds on selective medium. The resistant plants were transferred to soil and selfpollinated. Seeds were harvested for subsequent genetic, biochemical and molecular analysis.

\section{Acknowledgments}

We thank Dr Hsou-min Li for the in vitro chloroplast import assay and Dr Jane Langdale for reviewing the manuscript. S.-M.W. and J.C. were supported by the grants from the National Science Council, Taiwan, Republic of China (NSC 82-0418-B-002-376-B03 to S.-M.W. and NSC 84-2311-B-001-033 to J.C.) and Academia Sinica, Taipei, Taiwan (J.C.).

\section{References}

Bechtold, N., Ellis, J. and Pelletier, G. (1993) In planta Agrobacterium mediated gene transfer by infiltration of adult Arabidopsis thaliana plants. C. R. Acad. Sci. Paris, 316, 11941199.

Caspar, T., Huber, S.C. and Somerville, C. (1985) Alterations in growth, photosynthesis, and respiration in a starchless mutant of Arabidopsis thaliana $L$. deficient in a chloroplast phosphoglucomutase activity. Plant Physiol. 79, 11-17.

Chang, C., Bowman, J.L., DeJohn, A.W., Lander, E.S. and Meyerowitz, E.M. (1988) Restriction fragment length polymorphism map for Arabidopsis thaliana. Proc. Natl Acad. Sci. USA, 85, 6856-6860.

Copeland, L. and Preiss, J. (1981) Purification of spinach leaf ADPglucose pyrophosphorylase. Plant Physiol. 68, 996-1001.

Greene, T.W., Chantler, S.E., Kahn, M.L., Barry, G.F., Preiss, J. and Okita, T.W. (1996) Mutagenesis of the potato ADPglucose pyrophosphorylase and characterization of an allosteric mutant defective in 3-phosphoglycerate activation. Proc. Natl Acad. Sci. USA, 93, 1509-1513.

Haugen, T., Ishaque, A. and Preiss, J. (1976) Biosynthesis of bacterial glycogen. Characterization of the subunit structure of Escherichia coli B glucose-1-P adenylyl transferase. J. Biol. Chem. 251, 7880-7885.

Hylton, C. and Smith, A.M. (1992) The rb mutation of peas causes structural and regulatory changes in ADP glucose pyrophosphorylase form developing embryos. Plant Physiol. 99, 1626-1634.

Iglesias, A.A., Charng, Y.-y., Ball, S. and Preiss, J. (1994) Characterization of the kinetic, regulatory, and structural properties of ADP-glucose pyrophosphorylase from Chlamydomonas reinhardii. Plant Physiol. 104, 1287-1294.

Krishnan, H.B., Reeves, C.D. and Okita, T.W. (1986) ADPglucose pyrophosphorylase is encoded by different mRNA transcripts in leaf and endosperm of cereals. Plant Physiol. 81, 642-645.

Lin, T.-P., Caspar, T., Somerville, C. and Preiss, J. (1988a) Isolation and characterization of a starchless mutant of Arabidopsis thaliana (L.) Heynh lacking ADPglucose pyrophosphorylase activity. Plant Physiol. 86, 1131-1135. 
Lin, T.-P., Caspar, T., Somerville, C. and Preiss, J. (1988b) A starch deficient mutant of Arabidopsis thaliana with low ADPglucose pyrophosphate activity lacks one of the two subunits of the enzyme. Plant Physiol. 88, 1175-1181.

Li, L. and Preiss, J. (1992) Characterization of ADPglucose pyrophosphorylase from a starch-deficient mutant of Arabidopsis thaliana (L.). Carbohydrate Res. 227, 227-239.

Lister, C. and Dean, C. (1993) Recombinant inbred lines for mapping RFLP and phenotypic markers in Arabidopsis thaliana. Plant J. 4, 745-750.

Morell, M.K., Bloom, M., Knowles, V. and Preiss, J. (1987) Subunit structure of spinach leaf ADPglucose pyrophosphorylase. Plant Physiol. 85, 182-187.

Muller-Rober, B., Sonnewald, U. and Willmitzer, L. (1992) Inhibition of the ADP-glucose pyrophosphorylase in transgenic potatoes leads to sugar-storing tubers and influences tuber formation and expression of tuber storage protein genes. EMBO. J. 11, 1229-1238.

Nam, H.-G., Giraudat, J., den Boer, B., Moonan, F., Loos, W., Hauge, B. and Goodman, H.M. (1989) Restriction fragment length polymorphism linkage map of Arabidopsis thaliana. Plant Cell, 1, 699-705.

Preiss, J. (1988) Biosynthesis of starch and its regulation. In The Biochemistry of Plants (Preiss, J., ed.). New York: Academic Press, pp. 181-254.
Preiss, J. (1991) Biology and molecular biology of starch synthesis and its regulation. In Oxford Surveys of Cellutar and Molecular Biology, Volume 7 (Miflin, B.J., ed.). Oxford: Oxford University Press, pp. 59-114.

Sambrook, J., Fritsh, E.F. and Maniatis, T. (1989) Molecular Cloning. 2nd Edn. Cold Spring Harbor, NY: Cold Spring Harbor Laboratory Press.

Smith, A.M., Bettey, M. and Bedford, I.D. (1989) Evidence that the $r b$ locus alters the starch content of developing pea embryos through an effect on ADP glucose pyrophosphorylase. Plant Physiol. 89, 1279-1284.

Smith-White, B.J. and Preiss, J. (1992) Comparison of proteins of ADP-glucose pyrophosphorylase from diverse sources. J. Mol. Evol. 34, 449-464.

Stam, P. (1993) Construction of integrated genetic linkage maps by means of a new computer package: JoinMap. Plant J. 3, 739-744.

Villand, P., Olsen, O-A. and Kleczkowski, L.A. (1993) Molecular characterization of multiple CDNA clones for ADP-glucose pyrophosphorylase from Arabidopsis thaliana. Plant Mol. Biol. 23, 1279-1284.

Weaver, S.H., Glover, D.V. and Tsai, C.Y. (1972) Nucleoside diphosphate glucose pyrophosphorylase isozymes of developing normal, brittle-2, and shrunken-2 endosperms of Zea mays L. Crop Sci. 12, 510.

The GenBank Data Library accession number U72290 (ADPG pyrophosphorylase large subunit cDNA sequence). 